\title{
Cisplatin-induced regulation of signal transduction pathways and transcription factors in p53-mutated subclone variants of hepatoma cells: Potential application for therapeutic targeting
}

\author{
JINN-RUNG KUO ${ }^{1,2 *}$, HUNG-SHENG SHANG ${ }^{3 *}$, CHUN-TE HO $^{4}$, KUN-GOUNG LAI ${ }^{5}$, \\ TSAN-ZON LIU ${ }^{6}$, YIN-JU CHEN ${ }^{7-9}$ and JENG-FONG CHIOU 7,10
}

\begin{abstract}
Departments of ${ }^{1}$ Neurosurgery and ${ }^{2}$ Medical Research, Chi-Mei Medical Center, Tainan 71004;
${ }^{3}$ Division of Clinical Pathology, Department of Pathology, National Defense Medical Center, Tri-Service General Hospital, Taipei 11490; ${ }^{4}$ Graduate Institute of Medical Sciences, College of Medicine, Taipei Medical University, Taipei 11031;

${ }^{5}$ Department of Radiation Oncology, Tungs' Taichung Metro Harbor Hospital, Taichung 43503;

${ }^{6}$ Translational Research Laboratory, Cancer Center, Taipei Medical University; ${ }^{7}$ Department of Radiation Oncology,

Taipei Medical University Hospital; ${ }^{8}$ Graduate Institute of Biomedical Materials and Tissue Engineering,

College of Biomedical Engineering; ${ }^{9}$ Graduate Institute of Cancer Biology and Drug Discovery,

College of Medical Science and Technology; ${ }^{10}$ Department of Radiology, School of Medicine,

College of Medicine, Taipei Medical University, Taipei 11031, Taiwan, R.O.C.
\end{abstract}

Received May 19, 2015; Accepted August 9, 2016

DOI: $10.3892 / 01.2016 .5181$

\begin{abstract}
Cisplatinis commonly recognized as aDNA-damaging drug; however, its versatile antitumor effects have been demonstrated to extend beyond this narrow functional attribute. The present study determined how cisplatin regulates alternative pathways and transcription factors to exert its additional antitumor actions. Cisplatin was observed to be able to trigger an endoplasmic reticulum stress response through aggravated nitrosative stress coupled to perturbed mitochondrial calcium $\left(\mathrm{Ca}^{2+}\right)$ homeostasis, which substantially downregulated glucose-regulated protein (GRP) 78 expression by suppressing the cleavage of activating transcription factor (ATF) $6 \alpha(90 \mathrm{kDa})$ to its active $50 \mathrm{kDa}$ subunit. Concomitantly, the ATF4-ATF3-C/emopamil binding protein homologous protein axis was activated by cisplatin, which triggered cellular glutathione (GSH) depletion by strongly inhibiting $\gamma$-glutamylcysteine synthetase heavy chain $\left(\gamma-\mathrm{GCS}_{\mathrm{h}}\right)$, a key enzyme in GSH biosynthesis. The present study also demonstrated that cisplatin substantially inhibited $\beta$-catenin, causing a marked downregulation of survivin and B-cell lymphoma (Bcl)-2. Taken together, the present results
\end{abstract}

Correspondence to: Professor Jeng-Fong Chiou or Dr Yin-Ju Chen, Department of Radiation Oncology, Taipei Medical University Hospital, $250 \mathrm{Wu}-\mathrm{H}$ sing Street, Taipei 11031, Taiwan, R.O.C.

E-mail: solomanc@tmu.edu.tw

E-mail: yjchen1113@gmail.com

*Contributed equally

Key words: cisplatin, GSH depletion, ER stress, GRP78, survivin, Bcl-2 uncovered a novel mechanism of cisplatin that could simultaneously trigger the inhibition of three prominent antiapoptotic effector molecules (Bcl-2, survivin and GRP78) and effectively promote GSH depletion by inhibiting $\gamma-\mathrm{GCS}_{\mathrm{h}}$. These newly discovered functional attributes of cisplatin can provide an avenue for novel combined therapeutic strategies to kill hepatocellular carcinoma cells effectively.

\section{Introduction}

Among men, hepatocellular carcinoma (HCC) is the fifth most common cancer worldwide and the second leading cause of cancer-associated mortalities annually (1). Current curative treatments, including surgical resection and liver transplantation, are generally ineffective, and can be applied only during early-stage HCC $(2,3)$. In addition, only $10-15 \%$ of patients are qualified to be treated with curative surgery $(2,3)$. Thus, the majority of $\mathrm{HCC}$ patients must be considered for systemic chemotherapies or supportive therapies (3). However, the majority of chemotherapeutic agents exhibit poor effectiveness and can only marginally improve patient survival rates (3).

Cisplatin is one of the most widely used chemotherapeutic agents for treating various types of malignancies (4). Cisplatin is primarily considered as a DNA-damaging agent that forms various types of bifunctional adducts upon reacting with cellular DNA (4). The final cellular outcome of DNA adduct formation is generally apoptotic cell death, which is considered to occur through the disruption of cellular processes such as the deregulation of signal transduction pathways involved in growth, differentiation and stress responses (5). Although DNA damage is widely considered to be responsible for cisplatin-induced apoptosis, the molecular mechanisms that establish the formation of DNA adducts in response to 
cisplatin-induced apoptosis have not been identified conclusively $(6,7)$. This research gap raises the question of whether alternative and/or additional mechanisms are involved. Accumulating evidence has revealed that cisplatin-induced apoptosis could occur independently of DNA damage through oxidative stress in various cell types (8-10). Therefore, alternative pathways affected by cisplatin should be further explored, since they could yield critical insights for improving the current cisplatin-based regimens. Despite the fact that the current postulated mechanisms remain controversial, there is a leading theory that involves the ability of cisplatin to inhibit sublethal damage repair in resistant tumor cells $(5,6)$. Thus, further characterization of the possible underlying mechanism(s) associated with the sensitization engendered by cisplatin in HCC cells is justified.

With these premises in mind, the aim of the present study was to identify the molecular signatures of selected HCC cell lines and to provide mechanistic insight into apoptotic pathways alternatively activated by cisplatin to elucidate the fundamental causes of sensitization during endoplasmic reticulum (ER) stress. In the present study, two p53-dysfunctional HCC models, Hep3B and Mahlavu (11), were selected, of which, the former was more differentiated than the latter. It was noticed that Mahlavu cells were distinctly more sensitive to cisplatin than Hep3B cells. Our results also revealed a novel functional attribute of cisplatin involving the robust suppression of two prominent pathways that regulate the expression of B-cell lymphoma (Bcl)-2, survivin and glucose-regulated protein (GRP) 78 independently of DNA damage and repair mechanisms. Thus, these findings provide a crucial missing link in the understanding of the mechanism of cisplatin-induced ER stress. Critically, this novel functional attribute of cisplatin may also provide an avenue for novel therapeutic strategies against chemotherapy-resistant HCC cells.

\section{Materials and methods}

Cell culture and cell viability assay. The two HCC cell lines used in the present study, Hep3B cells purchased from Bioresource Collection and Research Center (Hsinchu, Taiwan) and Mahlavu cells provided by Professor K.H. Lin (Chang Gung University, Taiwan), were grown in Dulbecco's modified Eagle's medium (Invitrogen; Thermo Fisher Scientific, Inc., Waltham, MA, USA) supplemented with $10 \%$ (v/v) fetal bovine serum (Biological Industries, Cromwell, CT, USA) in $5 \% \mathrm{CO}_{2}$, in a humidified incubator $\left(37^{\circ} \mathrm{C}\right)$. The cells were treated with cisplatin (Sigma-Aldrich; Merck Millipore, Darmstadt, Germany) at various concentrations for 24 or $72 \mathrm{~h}$, and cell viability was measured using a sulforhodamine B (SRB) assay, as previously described (12). Briefly, cells were fixed using trichloroacetic acid and then incubated with SRB (Sigma-Aldrich; Merck Millipore) upon being washed and air-dried. The precipitate was dissolved in Trizma ${ }^{\circledR}$ base solution (Sigma-Aldrich; Merck Millipore). The absorbance was measured at $540 \mathrm{~nm}$ by using a microplate reader.

Western blot analysis. A total of $5 \times 10^{5}$ cells were seeded for $24 \mathrm{~h}$ and treated with cisplatin for different periods. Cells were lysed in radioimmunoprecipitation assay buffer (Sigma-Aldrich; Merck Millipore), and protein concentrations were assayed using the Bio-Rad Protein Assay kit (Bio-Rad Laboratories, Inc., Hercules, CA, USA). In total, $40 \mu \mathrm{g}$ of proteins was separated on $12 \%$ sodium dodecyl sulfate-polyacrylamide gel electrophoresis and transferred electrophoretically to a polyvinylidene difluoride membrane. Upon blocking, the membrane was hybridized at $4^{\circ} \mathrm{C}$ overnight with one of the following primary antibodies: Anti-GRP78 (catalog no. sc-13968; dilution, 1:500), anti-activating transcription factor (ATF) 3 (catalog no. sc-188; dilution, 1:500), anti-ATF4 (catalog no. sc-200; dilution, 1:500), anti-ATF6 (50 kDa; catalog no. sc-22799; dilution, 1:500), anti-C/emopamil binding protein homologous protein (CHOP; (catalog no. sc-7351; dilution, 1:500), anti- $\gamma$-glutamylcysteine synthetase heavy chain $\left(\gamma-\mathrm{GCS}_{\mathrm{h}}\right.$; catalog no. sc-100747; dilution, 1:500), anti-Bcl-2 (ctalog no. sc-7382; dilution, 1:500), anti-survivin (catalog no. sc-10811; dilution, 1:500) (all Santa Cruz Biotechnology, Inc., Dallas, TX, USA), anti- $\beta$-catenin (catalog no. 06-734; dilution, 1:2,500; Upstate Biotechnology, Inc., Lake Placid, NY, USA), anti-poly(adenosine diphosphateribose) polymerase 1 (PARP-1; 1074-1; dilution, 1:1,000; Epitomics, Burlingame, CA, USA) and anti- $\beta$-actin (catalog no. A5441; dilution, 1:10,000; Sigma-Aldrich; Merck Millipore) antibodies, followed by incubation with secondary antibodies conjugated to horseradish peroxidase. The antigen-antibody complexes were detected using a Pierce enhanced chemiluminescence detection system (Thermo Fisher Scientific, Inc.).

Flow cytometric measurement of intracellular nitric oxide (NO) and cellular glutathione (GSH) depletion. All fluorescence reagents were purchased from Sigma-Aldrich (Merck Millipore), unless otherwise specified. Intracellular NO production was measured using 4,5-diaminofluorescein (DAF-2), as described previously (13). Briefly, cells were treated with cisplatin, washed with phosphate-buffered saline (PBS) and then incubated with $1 \mu \mathrm{M}$ DAF-2 for $10 \mathrm{~min}$ in the dark. The cells were then washed with PBS, detached by trypsinization, collected by centrifugation at $0.2 \mathrm{x} g$ at room temperature and resuspended in PBS. To measure cellular GSH depletion, cisplatin-treated cells were incubated with $25 \mu \mathrm{M}$ 5-chloromethylfluorescein (CMF) diacetate for $20 \mathrm{~min}$ at $5 \% \mathrm{CO}_{2}$ in a $37^{\circ} \mathrm{C}$ incubator. The CMF fluorescence intensity was measured using a BD FACSCalibur ${ }^{\mathrm{TM}}$ flow cytometer (version 3.3; BD Biosciences, San Jose, CA, USA) and analyzed using BD CellQuest Pro software (BD Biosciences).

Detecting intracellular NO using confocal microscopy. To measure the production of intracellular NO, cisplatin- and sham-treated HCC cells were cultured in poly-L-lysine-coated slides. All fluorescence reagents were purchased from Sigma-Aldrich (Merck Millipore). Upon reaching $80 \%$ cell density, the cells were incubated with $2 \mu \mathrm{M}$ DAF-2',7'-difluorofluorescein diacetate in 4-(2-hydroxyethyl)-1-piperazineethanesulfonic acid for 30 min in the dark and imaged using a Leica TCS SP2 laser scanning confocal microscope (Leica Microsystems GmbH, Wetzlar, Germany).

Confocal microscopic detection of mitochondrial and cytosolic calcium $\left(\mathrm{Ca}^{2+}\right)$. Cells were stained with $1.5 \mu \mathrm{M}$ Rhod-2 and $2 \mathrm{mM}$ Fluo-4 (Invitrogen; Thermo Fisher Scientific, Inc.) 
A
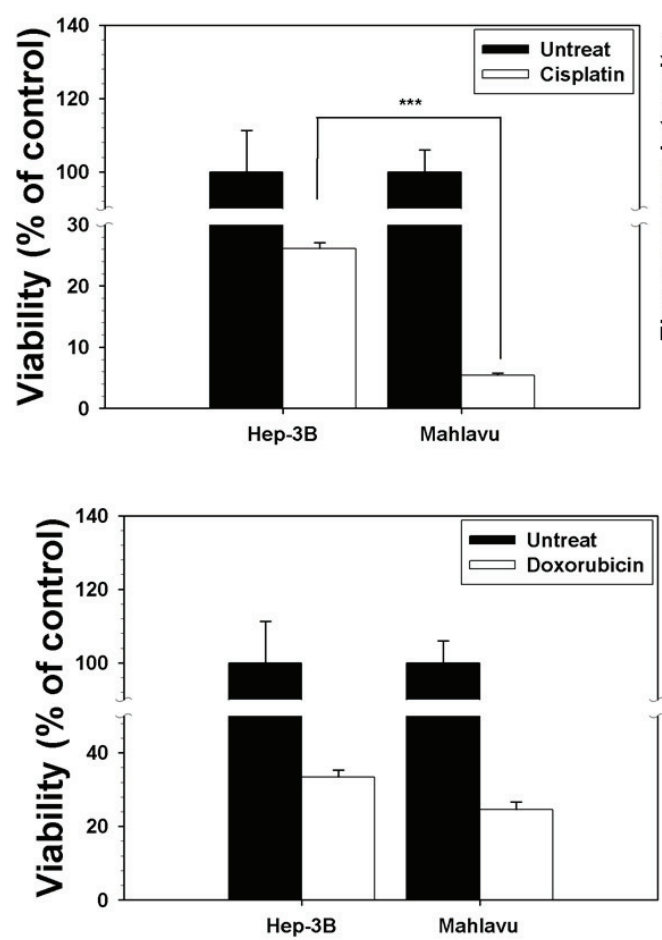

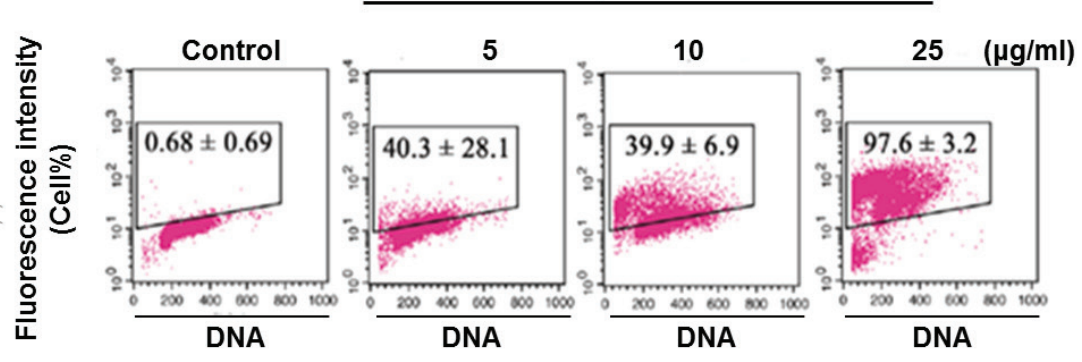

C

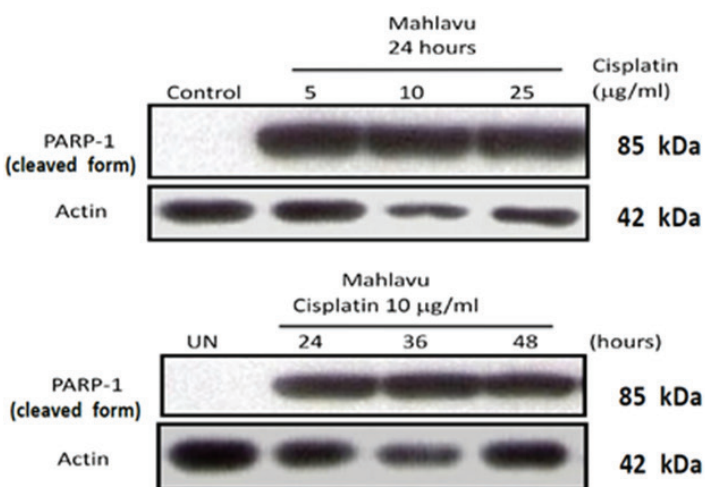

Figure 1. Distinct disparity of sensitivity of hepatocellular carcinoma cells in response to chemotherapeutic drugs. (A) Mahlavu and Hep3B cells were treated with cisplatin or doxorubicin for $72 \mathrm{~h}$. Cell viability was determined using a sulforhodamine B assay. The results are expressed as the mean \pm standard deviation. Each point represents an average of $\geq 3$ determinations. ${ }^{* * * *} \mathrm{P}<0.05$ vs. control. (B) Evaluation of cisplatin-induced apoptosis in Mahlavu cells. Control (untreated) cells and cells treated with 5, 10 or $25 \mu \mathrm{g} / \mathrm{ml}$ cisplatin for $24 \mathrm{~h}$ were subjected to terminal deoxynucleotidyl transferase 2 -deoxyuridine 5'-triphosphate assay. The representative plots depict DNA content on the X axis and 5-bromo-2'-deoxyuridine-fluorescein isothiocyanate-labeled apoptotic DNA strand breaks on the Y axis. The data represent the percentage of apoptotic cells in the upper boxes. (C) The cleavage of PARP-1 (a 116-kDa nuclear enzyme downstream of caspase 3) into an 85-kDa fragment by cisplatin-treated cells provides further evidence of the occurrence of apoptosis through the activation of caspase 3. UN, untreated; PARP-1, poly(adenosine diphosphate-ribose) polymerase 1.

to detect mitochondrial and cytosolic $\mathrm{Ca}^{2+}$, respectively. Fluorescent images of Rhod-2 and Fluo-4 were obtained prior and subsequent to treating the cells with cisplatin. At the end of each experimental period, cells were mounted on the stage of a Leica TCS SP2 laser scanning confocal microscope. Then, cells were randomly selected and imaged.

Terminal deoxynucleotidyl transferase 2'-deoxyuridine 5'-triphosphate nick end labeling (TUNEL) assay. DNA fragmentation was detected using a TUNEL assay and an Apo-BrdU In Situ DNA Fragmentation Assay kit (BioVision, Inc., Milpitas, CA, USA), according to the manufacturer's protocol. A BD FACSCalibur ${ }^{\mathrm{TM}}$ was used to perform the analyses. DNA content was quantified using ModFit LT ${ }^{\mathrm{TM}}$ software (version 3.0; Verity Software House, Inc., Topsham, ME, USA).

Statistical analysis. All data are presented as the mean \pm standard deviation from $\geq 3$ independent experiments, and were analyzed using the Student's t-test. $\mathrm{P}<0.05$ was considered to indicate a statistically significant difference.

\section{Results}

HCC cells exhibit distinct disparity in response to chemotherapeutic drugs. Two chemotherapeutic drugs, cisplatin and doxorubicin, were selected to test their cytotoxicity effect on two HCC cell lines, Mahlavu (a poorly differentiated and highly Bcl-2-expressing cell line with a p53 mutation at codon 249) and Hep3B (a well-differentiated and low Bcl-2-expressing subline formed by p53-null cells) (13-15). As indicated in Fig. 1A, upon treatment with cisplatin, cell viability decreased to $5 \%$ in the Mahlavu cells, whereas $28 \%$ of the Hep3B cells remained viable. However, there was no significant difference between the Mahlavu and Hep3B cells upon treatment with doxorubicin $(\mathrm{P}=0.086)$. By contrast, the Mahlavu cells were observed to be distinctly more sensitive to cisplatin than Hep3B cells. Next, cisplatin-induced apoptosis was investigated by a TUNEL assay. Cisplatin induced cell apoptotic death in a concentrationdependent manner in the Mahlavu cells (Fig. 1B), and it also induced the cleavage of PARP-1 (a 116-kDa nuclear enzyme downstream of caspase 3) to produce an $85-\mathrm{kDa}$ fragment (Fig. 1C). These data clearly demonstrated that the cell death associated with cisplatin treatment was apoptotic in nature.

Cisplatin provokes $\mathrm{NO}$ production and perturbs $\mathrm{Ca}^{2+}$ homeostasis. Accumulating evidence indicates that cisplatininduced apoptosis could occur independently of DNA damage through oxidative stress in various cell types (5-7). Therefore, it was examined whether cisplatin regulates ER stress through aggravated nitrosative stress coupled to perturbed mitochondrial $\mathrm{Ca}^{2+}$ homeostasis. As represented in Fig. 2A, when Mahlavu 
A

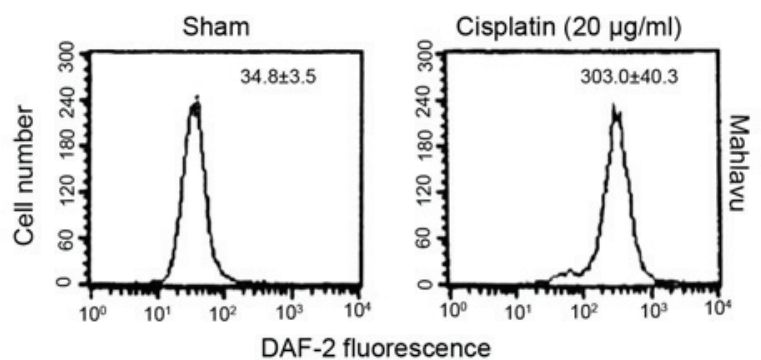

B

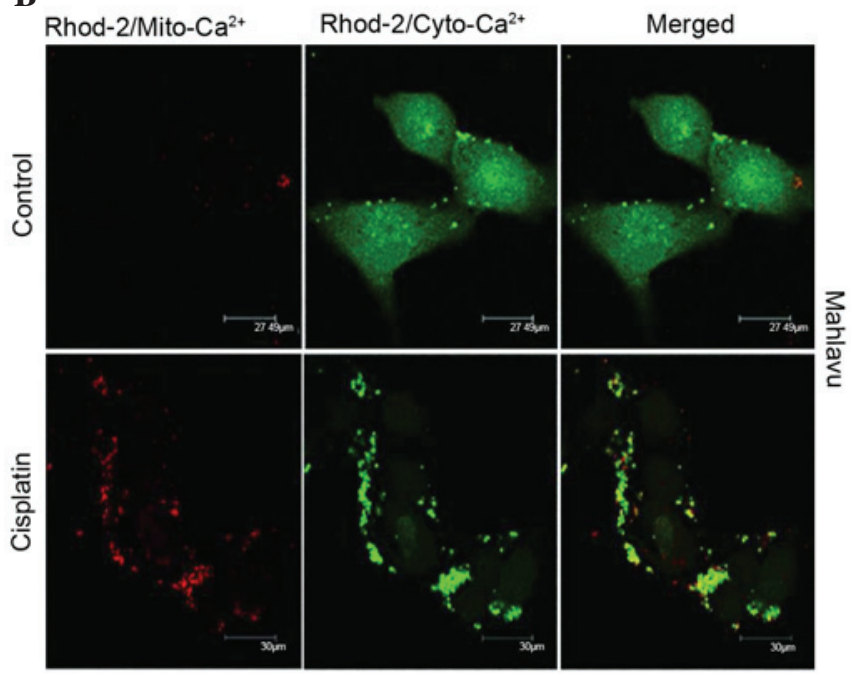

Figure 2. Cisplatin could provoke increased production of $\mathrm{NO}$ and perturbed $\mathrm{Ca}^{2+}$ homeostasis. (A) A nearly 10 -fold increase in NO production was observed when Mahlavu cells were treated with $20 \mu \mathrm{g} / \mathrm{ml}$ cisplatin, as demonstrated by flow cytometry using DAF-2 as a probe. (B) At resting state, Mahlavu cells exhibited relatively low levels of mitochondrial $\mathrm{Ca}^{2+}$ (Rhod-2/sham) (x400 magnification). Upon cisplatin treatment $(25 \mu \mathrm{g} / \mathrm{ml})$, significantly increased Rhod-2 fluorescence (mito- $\mathrm{Ca}^{2+}$ ) (red color) was detected, accompanied by a concomitantly reduced Fluo- 4 fluorescence (cyto- $\mathrm{Ca}^{2+}$ ) (green color), indicating that mitochondrial $\mathrm{Ca}^{2+}$ overload had occurred. DAF-2, 4,5-diaminofluorescein; $\mathrm{NO}$, nitric oxide; $\mathrm{Ca}^{2+}$, calcium Mito, mitochondrial; Cyto, cytosolic.

cells were treated with cisplatin, a stimulated overproduction of NO was observed, according to the data obtained using flow cytometry. Furthermore, cisplatin triggered an increased influx of cytosolic $\mathrm{Ca}^{2+}$ into the mitochondria and caused a $\mathrm{Ca}^{2+}$ overload in these organelles, as indicated by the red fluorescence of Rhod-2 (Fig. 2B). Thus, cisplatin was demonstrated to induce ER stress response through aggravated nitrosative stress coupled to perturbed mitochondrial $\mathrm{Ca}^{2+}$ homeostasis.

Cisplatin-induced ER stress response activates the ATF4ATF3-CHOP axis. The present study demonstrated that cisplatin-induced ER stress response could also regulate the ATF4-ATF3-CHOP axis and its downstream molecules. In Mahlavu cells treated with cisplatin for $24 \mathrm{~h}$, the ATF4-ATF3-CHOP axis was concomitantly activated following treatment (Fig. 3A). The CHOP-mediated downstream molecule GSH may be critical for cell apoptosis (16). In the present study, a concentration-dependent depletion of intracellular GSH was identified using flow cytometry (Fig. 3B). Additionally, the current results revealed the first evidence that CHOP-mediated GSH depletion is at least in part due to the strong inhibition by cisplatin of $\gamma-\mathrm{GCS}_{\mathrm{h}}$, a rate-limiting enzyme responsible for cellular GSH biosynthesis (Fig. 3C) (17).
Cisplatin severely downregulates GRP78 via suppressed ATF6 $\alpha$-p50 expression. ER stress can induce transcriptional factor activation; therefore, the present study sought to determine whether cisplatin-induced ER stress regulates the activation of the transcriptional factor ATF6 $\alpha$. At resting state, Mahlavu cells were observed to be a high GRP78-expressing HCC subline, due to its constitutive high levels of active ATF6 $\alpha$-p50. However, upon cisplatin treatment, strong suppression of the active ATF6 $\alpha$-p50 form and downregulation of GRP78 expression was observed (Fig. 4A and B).

Cisplatin induces survivin and Bcl-2 downregulation through inhibiting $\beta$-catenin. Since the availability of $\beta$-catenin and its nuclear translocation is key in controlling the transcription of downstream genes of the Wnt signaling pathway $(18,19)$, the current study investigated whether cisplatin could also downregulate the expression of survivin and $\mathrm{Bcl}-2$ through inhibiting $\beta$-catenin. The results revealed that the expression of survivin (Fig. 4C and D) and Bcl-2 (Fig. 4E and F) in the Mahlavu cells was substantially inhibited by cisplatin in a time- and concentration-dependent manner. This phenomenon correlated closely with the inhibition of $\beta$-catenin, suggesting that the expression of survivin and Bcl-2 through the Wnt signaling pathway was largely blocked.

\section{Discussion}

The present study identified a crucial missing link in the understanding of the mechanism associated with cisplatin-induced ER stress. Two HCC model cell lines were selected, Mahlavu and Hep3B, with different p53 dysfunctions. Generally, HCC lines were subjected to higher doses of cisplatin $(25-200 \mu \mathrm{M})$ in previous platinum-based drug studies $(20,21)$, according to the literature. For example, Hep3B was observed to be resistant to p53-mediated growth arrest and apoptosis (22), and received a high dose of cisplatin ( $60 \mu \mathrm{M}$ for $24 \mathrm{~h}$ ) in a previous study (20). Notably, when comparing the two HCC cell lines used in the present study, the Mahlavu cells were observed to be considerably more sensitive to cisplatin than the Hep3B cells (Fig. 1). This result may reflect differences in their p53 and molecule expression status $(14,15)$. The cisplatin response has been reported to involve ER stress $(8,23,24)$. The ER is the most critical protein-folding compartment and an intracellular $\mathrm{Ca}^{2+}$ storage organelle in cells (25). Thus, accumulating reactive nitrogen species could modify proteins and disrupt $\mathrm{Ca}^{2+}$ homeostasis, which may trigger the induction of cisplatin-induced ER stress $(26,27)$. Upon cisplatin treatment, intracellular NO levels increased nearly by 10 -fold in the Mahlavu cells (Fig. 2A). This increased NO production could have caused ER $\mathrm{Ca}^{2+}$ depletion and subsequently increased the influx of cytosolic $\mathrm{Ca}^{2+}$ into the mitochondria, which could eventually trigger mitochondrial $\mathrm{Ca}^{2+}$ overload (Fig. 2B). These findings support the notion that NO-dependent mitochondrial disruption can be coupled to the ER stress response $(14,15,20,21)$. The present study revealed that cisplatin-induced ER stress response simultaneously provoked two prominent pathways that may induce cells apoptosis. The key effectors of ER stress-signaling pathways may modulate cisplatin-induced cell death (27) through GRP78 (24) or $\beta$-catenin (28). Therefore, identifying those molecules that can be used to predict cisplatin 
A

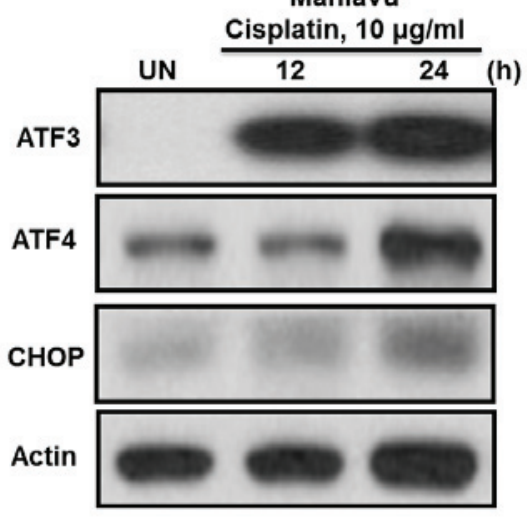

B

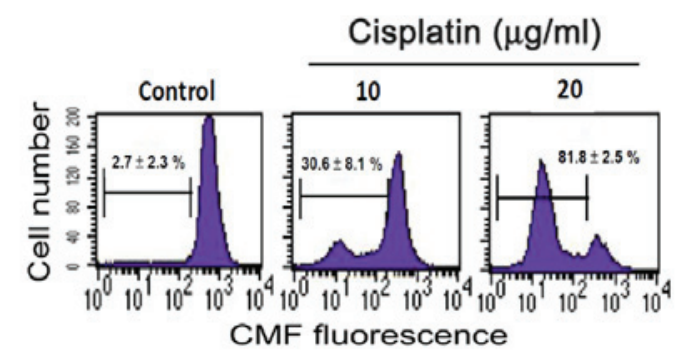

$\mathbf{C}$

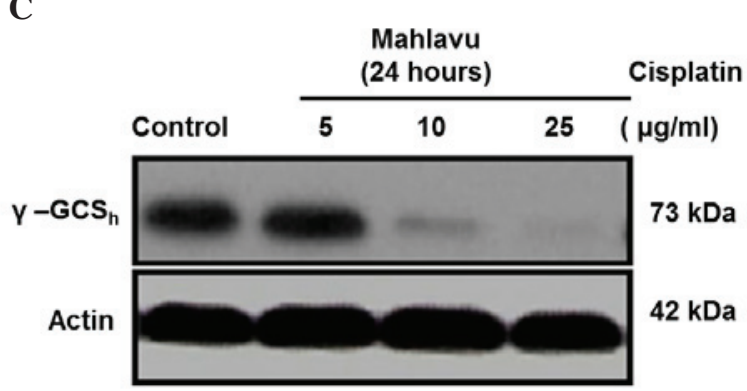

Figure 3. (A) Cisplatin could trigger an ER stress response in Mahlavu cells by activating the pro-apoptotic ATF4-ATF3-CHOP pathway. (B) The cisplatininduced ER stress response concomitantly caused cellular GSH depletion in a concentration-dependent manner. (C) The cisplatin-induced cellular GSH depletion was demonstrated to be due to a strong inhibition of $\gamma-\mathrm{GCS}_{\mathrm{h}}$, an enzyme involved in the regulation of GSH biosynthesis. ER, endoplasmic reticulum; ATF, activating transcription factor; CHOP, C/emopamil binding protein homologous protein; CMF, 5-chloromethylfluorescein; $\mathrm{GSH}_{\text {, glutathione; } \gamma \text {-GCS }}$, $\gamma$-glutamylcysteine synthetase heavy chain.

A

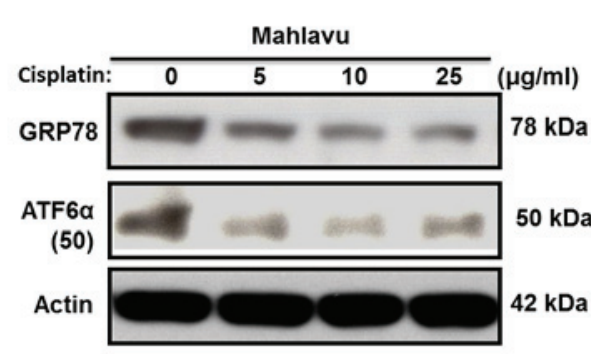

C

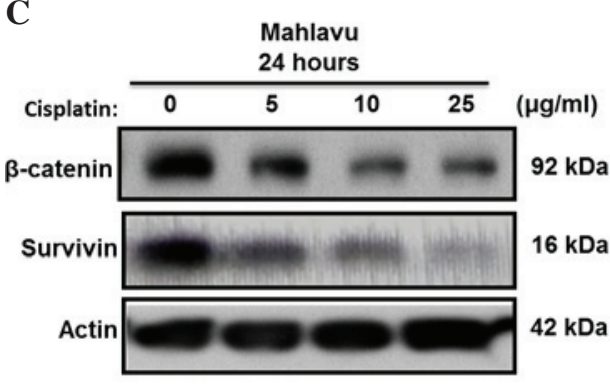

E

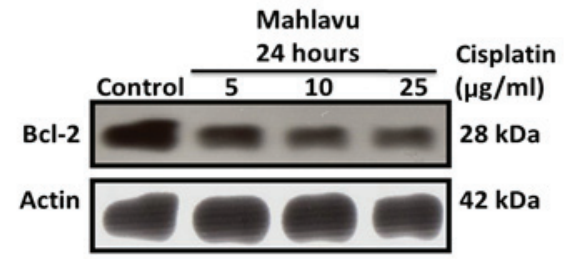

B

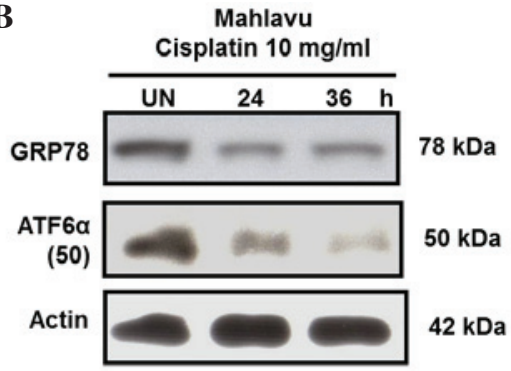

D

Mahlavu Cisplatin $10 \mathrm{mg} / \mathrm{ml}$

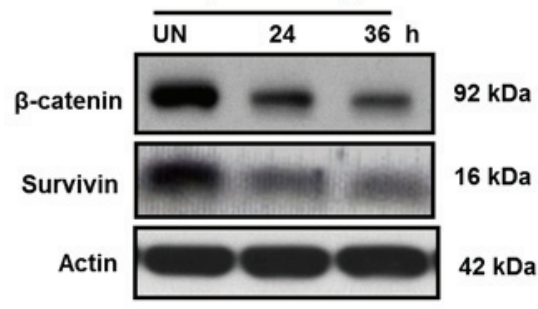

F

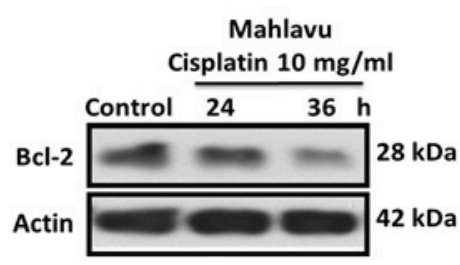

Figure 4. Inhibition of three prominent anti-apoptotic effectors by cisplatin and the underlying mechanisms involved. (A and B) Cisplatin concomitantly inhibited GRP78 and ATF6 $\alpha(50 \mathrm{kDa})$ in Mahlavu cells in a (A) concentration- and (B) time-dependent manner. (C and D) Cisplatin-treated Mahlavu cells exhibited suppressed expression of $\beta$-catenin and survivin, two major modifying molecules regulating anti-apoptotic signaling pathways, in a (C) concentrationand (D) time-dependent manner. Thus, (A and B) the blockade of ATF6 $\alpha(50 \mathrm{kDa})$ expression could suppress GRP78 production, whereas the suppression of $\beta$-catenin expression could perturb the Wnt signaling pathway and render (C and D) survivin and (E and F) Bcl-2 downregulation in a (E) concentration- and (F) time-dependent manner. ATF, activating transcription factor; GRP, glucose-regulated protein; Bcl-2, B-cell lymphoma 2. 


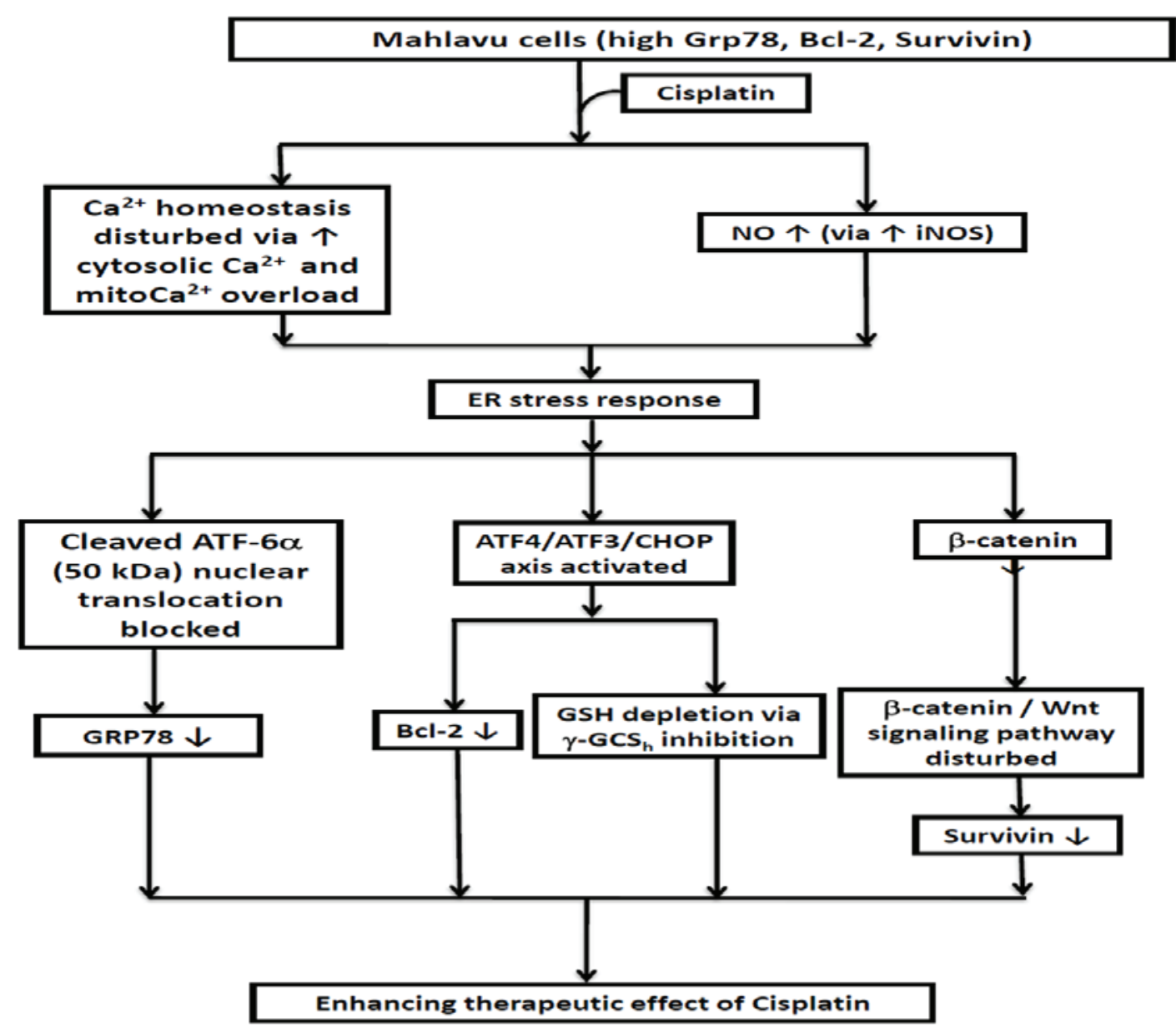

Figure 5. Diagrammatic illustration of the three critical cascades of events that negatively modulate the expression of three prominent cisplatin-associated anti-apoptotic effectors of hepatocellular carcinoma Mahlavu cells. RT, radiotherapy; $\mathrm{Ca}^{2+}$, calcium; NO, nitric oxide; iNOS, inducible nitric oxide synthase; ER, endoplasmic reticulum; ATF, activating transcription factor; CHOP, C/emopamil binding protein homologous protein; CMF, 5-chloromethylfluorescein; GSH, glutathione; $\gamma$-GCS,$\gamma$-glutamylcysteine synthetase heavy chain; GRP, glucose-regulated protein; Bcl-2, B-cell lymphoma 2.

treatment response would enable therapy to be tailored on an individual HCC patient basis. GRP78 is an ER-resident protein responsible for protein folding and assembly, and its expression level has been correlated with the acquisition of resistance by certain malignancies against diverse anticancer drugs $(29,30)$. Since the upregulation of GRP78 was frequently observed in HCC cells (15), the present study investigated how cisplatin affects the expression of GRP78 in association with the possible nitrosative stress-mediated mechanism in the Mahlavu cell model. The uniqueness of the Mahlavu cells was that they constitutively overexpressed GRP78, likely through a mild, endogenously generated NO-mediated increase in the active p50 form of the transcription factor ATF6 (ATF6 $\alpha$-p50), which, by nuclear translocation, could release GRP78 from its conjugated form $(18,26,27,31)$. In the present study, cisplatin was observed to suppress GRP78 through a mechanism involving the strong inhibition of the expression of ATF6 $\alpha$-p50. In this situation, the ability of the cells to withstand ER stress-induced apoptosis could then be considerably weakened. The present results also revealed that ATF4, ATF3 and CHOP were all highly induced by cisplatin (Fig. 3A). Cisplatin-induced CHOP expression is mediated through the cooperative interaction of ATF4 and ATF3 (Fig. 3A). Since ATF3 has been reported to be induced by a variety of stress-causing agents, including DNA damages (25), it is expected that cisplatin, a chemotherapeutic agent regarded as a typical DNA-damaging agent (Fig. 3A), could induce ATF3 expression. The upregulation of ATF3 has been demonstrated to play a pivotal role in the apoptosis induced by various histone deacetylase inhibitors and certain chemotherapeutic agents such as cisplatin $(32,33)$. Based on these observations, it can be concluded that the suppressed active ATF6 $\alpha$-p50 formed by cisplatin can render GRP78 downregulation. In this situation, the induction of CHOP mediated through the cross-talk of ATF4 and ATF3 can be triggered (31). Consistent with the findings reported in the literature, the present authors previously observed that cisplatin-induced ER stress response and CHOP expression could trigger GSH depletion $(31,34)$. Whether cisplatin-induced CHOP induction can also be triggered by a similar mechanism is currently under investigation by the present authors. Furthermore, the mechanism by which GSH depletion occurs during ER stress-mediated CHOP induction has not previously been addressed. The present study provides the first evidence that $\mathrm{CHOP}-$ mediated cellular 
GSH depletion is primarily due to the robust inhibition by cisplatin of $\gamma-\mathrm{GCS}_{\mathrm{h}}$, a rate-limiting enzyme responsible for the synthesis of cellular GSH (Fig. 2C) (17).

The activation of the Wnt/ $\beta$-catenin pathway has been reported in HCC cells $(18,19)$. $\beta$-catenin, a central effector molecule, works with the T-cell factor family of transcription factors to activate the expression of specific oncogenes, including cyclin D1, c-Myc, vascular endothelial growth factor, Bcl-2 and survivin (35-37). Mechanistically, determining the extent to which cisplatin is involved in modulating the transcriptional activity of the Wnt/ $\beta$-catenin signaling pathway that could eventually lead to the downregulation of survivin is warranted. Using confocal microscopy imaging, the present study determined that the majority of $\beta$-catenin was localized in the nucleus, thus leading to an increased expression of survivin (data not shown). Since $\beta$-catenin was overexpressed in Mahlavu cells, the present study observed that, upon cisplatin treatment, the $\beta$-catenin expression of the Mahlavu cells was substantially suppressed. This suppression could in turn markedly reduce the nuclear translocation of $\beta$-catenin, thus leading to the suppressed oncogenic expression of survivin (Fig. 4C and D) and Bcl-2 (Fig. 4E and F).

In conclusion, the present study provides the first evidence that the cisplatin-triggered apoptosis of HCC Mahlavu cells involves the robust inhibition of three prominent effector molecules, including GRP78, Bcl-2 and survivin, through the dualistic modulation of nitrosative stress-mediated ER stress response via activation of the ATF4-ATF3-CHOP axis and the downregulation of the $\mathrm{Wnt} / \beta$-catenin signaling pathway (Fig. 5). The current results suggest that cisplatin could be clinically useful in eradicating cancer cells that are resistant to chemotherapeutic agents due to the enhanced expression of GRP78, Bcl-2 or survivin.

\section{Acknowledgements}

The present study was supported by grants from the Taiwan National Science Council (Taipei, Taiwan; grant no. NSC 99-2314-B-038-026-MY3), the Ministry of Science and Technology of the R.O.C (Tapei, Taiwan; grant no. MOST 103-2314-B-038-058) and a Chi Mei Medical Center (CM) - Taipei Medical University (TMU) joint grant (Taipei, Taiwan; grant no. 98 CM-TMU 01-02).

\section{References}

1. Jemal A, Bray F, Center MM, Ferlay J, Ward E and Forman D: Global cancer statistics. CA Cancer J Clin 61: 69-90, 2011.

2. Lim YS, Han S, Heo NY, Shim JH, Lee HC and Suh DJ: Mortality, liver transplantation, and hepatocellular carcinoma among patients with chronic hepatitis B treated with entecavir vs lamivudine. Gastroenterology 147: 152-161, 2014.

3. Tabrizian P, Roayaie S and Schwartz ME: Current management of hepatocellular carcinoma. World J Gastroenterol 20: 10223-10237, 2014.

4. Kelland L: The resurgence of platinum-based cancer chemotherapy. Nat Rev Cancer 7: 573-584, 2007.

5. Stewart DJ: Mechanisms of resistance to cisplatin and carboplatin. Crit Rev Oncol Hematol 63: 12-31, 2007.

6. Vaisman A, Varchenko M, Said I and Chaney SG: Cell cycle changes associated with formation of Pt-DNA adducts in human ovarian carcinoma cells with different cisplatin sensitivity. Cytometry 27: 54-64, 1997.
7. Burger H, Nooter K, Boersma AW, Kortland CJ and Stoter G: Lack of correlation between cisplatin-induced apoptosis, p53 status and expression of Bcl-2 family proteins in testicular germ cell tumour cell lines. Int J Cancer 73: 592-599, 1997.

8. Mandic A, Hansson J, Linder S and Shoshan MC: Cisplatin induces endoplasmic reticulum stress and nucleus-independent apoptotic signaling. J Biol Chem 278: 9100-9106, 2003.

9. Baek SM, Kwon CH, Kim JH, Woo JS, Jung JS and Kim YK: Differential roles of hydrogen peroxide and hydroxyl radical in cisplatin-induced cell death in renal proximal tubular epithelial cells. J Lab Clin Med 142: 178-186, 2003.

10. Huang Y, Zhou S, Qui L, Wu J and Xu C: Effects of zinc gluconate on nephrotoxicity and glutathione metabolism disorder induced by cis-platin in mice. Drug Metabol Drug Interact 14: 41-46, 1997.

11. Kuo TC, Chang PY, Huang SF, Chou CK and Chao CC: Knockdown of HURP inhibits the proliferation of hepacellular carcinoma cells via downregulation of gankyrin and accumulation of p53. Biochem Pharmacol 83: 758-768, 2012.

12. Vichai V and Kirtikara K: Sulforhodamine B colorimetric assay for cytotoxicity screening. Nat Protoc 1: 1112-1116, 2006.

13. Chen CY, Liu TZ, Liu YW, Tseng WC, Liu RH, Lu FJ, Lin YS, Kuo SH and Chen CH: 6-shogaol (alkanone from ginger) induces apoptotic cell death of human hepatoma p53 mutant Mahlavu subline via an oxidative stress-mediated caspase-dependent mechanism. J Agric Food Chem 55: 948-954, 2007.

14. Matouk IJ, Mezan S, Mizrahi A, Ohana P, Abu-Lail R, Fellig Y, Degroot N, Galun E and Hochberg A: The oncofetal H19 RNA connection: Hypoxia, p53 and cancer. Biochim Biophys Acta 1803: 443-451, 2010

15. Wu CH, Uen YH, Ho CT, Tseng YT, Liu TZ, Chiou JF and Leung SW: Constitutive overexpression of Bcl-2, Survivin and ER stress chaperone GRP-78 confers intrinsic radioresistance in human hepatocellular carcinoma cells: Insight into the mechanistic pathways involved". J Cancer Ther 4: 399, 2013.

16. Hsu HC, Chiou JF, Wang YH, Chen CH, Mau SY, Ho CT, Change PJ, Liu TZ and Chen CH: Folate deficiency triggers an oxidative-nitrosative stress-mediated apoptotic cell death and impedes insulin biosynthesis in RINm5F pancreatic islet beta-cells: Relevant to the pathogenesis of diabetes. PLoS One 8: e77931, 2013.

17. Griffith OW and Meister A: Potent and specific inhibition of glutathione synthesis by buthionine sulfoximine (S-n-butyl homocysteine sulfoximine). J Biol Chem 254: 7558-7560, 1979.

18. Lee HC, Kim M and Wands JR: Wnt/Frizzled signaling in hepatocellular carcinoma. Front Biosci 11: 1901-1915, 2006.

19. Takigawa Y and Brown AM: Wnt signaling in liver cancer. Curr Drug Targets 9: 1013-1024, 2008.

20. Kim Y, Jang M, Lim S, Won H, Yoon KS, Park JH, Kim HJ, Kim BH, Park WS, Ha J and Kim SS: Role of cyclophilin B in tumorigenesis and cisplatin resistance in hepatocellular carcinoma in humans. Hepatology 54: 1661-1678, 2011.

21. Lim SC, Choi JE, Kang HS and Han SI: Ursodeoxycholic acid switches oxaliplatin-induced necrosis to apoptosis by inhibiting reactive oxygen species production and activating $\mathrm{p} 53$-caspase 8 pathway in HepG2 hepatocellular carcinoma. Int J Cancer 126: 1582-1595, 2010.

22. Friedman SL, Shaulian E, Littlewood T, Resnitzky D and Oren M: Resistance to p53-mediated growth arrest and apoptosis in Hep 3B hepatoma cells. Oncogene 15: 63-70, 1997.

23. Rabik CA, Fishel ML, Holleran JL, Kasza K, Kelley MR, Egorin MJ and Dolan ME: Enhancement of cisplatin [cis-diammine dichloroplatinum (II)] cytotoxicity by O6-benzylguanine involves endoplasmic reticulum stress. J Pharmacol Exp Ther 327: 442-452, 2008

24. Ahmad M, Hahn IF and Chatterjee S: GRP78 up-regulation leads to hypersensitization to cisplatin in A549 lung cancer cells. Anticancer Res 34: 3493-3500, 2014.

25. Xu W, Liu L, Charles IG and Moncada S: Nitric oxide induces coupling of mitochondrial signalling with the endoplasmic reticulum stress response. Nat Cell Biol 6: 1129-1134, 2004.

26. Zhao S, Xiong Z, Mao X, Meng D, Lei Q, Li Y, Deng P, Chen M, $\mathrm{Tu} \mathrm{M}, \mathrm{Lu} \mathrm{X}$, et al: Atmospheric pressure room temperature plasma jets facilitate oxidative and nitrative stress and lead to endoplasmic reticulum stress dependent apoptosis in HepG2 cells. PLoS One 8: e73665, 2013.

27. Xu Y, Wang C and Li Z: A new strategy of promoting cisplatin chemotherapeutic efficiency by targeting endoplasmic reticulum stress. Mol Clin Oncol 2: 3-7, 2014.

28. Verras M, Papandreou I, Lim AL and Denko NC: Tumor hypoxia blocks Wnt processing and secretion through the induction of endoplasmic reticulum stress. Mol Cell Biol 28: 7212-7224, 2008. 
29. Chiu CC, Lee LY, Li YC, Chen YJ, Lu YC, Li YL, Wang HM, Chang JT and Cheng AJ: Grp78 as a therapeutic target for refractory head-neck cancer with CD24(-)CD44(+) stemness phenotype. Cancer Gene Ther 20: 606-615, 2013.

30. Lin JA, Fang SU, Su CL, Hsiao CJ, Chang CC, Lin YF and Cheng CW: Silencing glucose-regulated protein 78 induced renal cell carcinoma cell line G1 cell-cycle arrest and resistance to conventional chemotherapy. Urol Oncol 32: 29.e1-e11, 2014.

31. St Germain C, O'Brien A and Dimitroulakos J: Activating Transcription Factor 3 regulates in part the enhanced tumour cell cytotoxicity of the histone deacetylase inhibitor M344 and cisplatin in combination. Cancer Cell Int 10: 32, 2010.

32. Yang N, Zhang H, Si-Ma H, Fu Y, Zhao W, Li D and Yang G: Dexamethasone decreases hepatocellular carcinoma cell sensitivity to cisplatin-induced apoptosis. Hepatogastroenterology 58 1730-1735, 2011.

33. Liu G, Su L, Hao X, Zhong N, Zhong D, Singhal S and Liu X: Salermide up-regulates death receptor 5 expression through the ATF4-ATF3-CHOP axis and leads to apoptosis in human cancer cells. J Cell Mol Med 16: 1618-1628, 2012.
34. Dong D, Ni M, Li J, Xiong S, Ye W, Virrey JJ, Mao C, Ye R, Wang M, Pen L, et al: Critical role of the stress chaperone GRP78/BiP in tumor proliferation, survival, and tumor angiogenesis in transgene-induced mammary tumor development. Cancer Res 68: 498-505, 2008.

35. Altieri DC: Molecular circuits of apoptosis regulation and cell division control: The survivin paradigm. J Cell Biochem 92: 656-663, 2004

36. Tien LT, Ito M, Nakao M, Niino D, Serik M, Nakashima M, Wen CY, Yatsuhashi $\mathrm{H}$ and Ishibashi $\mathrm{H}$ : Expression of beta-catenin in hepatocellular carcinoma. World J Gastroenterol 11: 2398-2401, 2005.

37. Thompson MD and Monga SP: WNT/beta-catenin signaling in liver health and disease. Hepatology 45: 1298-1305, 2007. 